\title{
Proposal of System to Prevent Midnight Prowl
}

\author{
Yuhki Takahashi $^{\mathrm{a}}$, Shota Nakashima ${ }^{\mathrm{b}}$, Yuhki Kitazono ${ }^{\mathrm{a},{ }^{*}}$ \\ ${ }^{a}$ Kitakyushu National College of Technology,5-20-1 Shii, Kokuraminami-ku, Kitakyushu-city, \\ Fukuoka 802-0985, Japan \\ bYamaguchi University, 2-16-1 Tokiwadai, Ube-city, Yamaguchi755-8611, Japan \\ *Corresponding Author:kitazono@kct.ac.jp
}

\begin{abstract}
In this study, we developed system to prevent midnight prowl using the depth camera and checked the operation of the system constructed. First, installed the camera on the ceiling, get depth information in the field of view, and also got background's depth information. The detection of human get up was performed by taking the difference between the depth and the current depth of the background. Converted into numeric depth information a distance from the camera to be retrieved as a string, and obtains the height of the object from the difference between them. Take a threshold value than the height that was acquired to detect anything more than a certain height, and also in after removing those narrow areas coordinates from the coordinates, and performs the tracking to obtain the center coordinates of the subject. We went from the center point when the track is interrupted the entry and exit detection.
\end{abstract}

Keywords: Welfare, Sensor, Depth information

\section{Introduction}

Today in Japan, the proportion of elderly people has been increasing due to an aging society, it has caused a society that is needed even more their caregivers ${ }^{(1)}$. In particular, late-night loitering in the elderly may be mentioned as one of the problems in the field of welfare. For the risk of an accident resulting in injury or late at night wandering, caregivers need a great deal of mental effort in the crisis management ${ }^{(2)}$. There are existing midnight prowl prevention systems to solve these. For example, mat sensor type, tag sensor type, sensor pad type, and infrared sensor. But these has problem each. A problem of the mat sensor is that it would be able to pass it easily avoided. A problem of tag sensor is that the subject may take off the tag. A problem of the pad sensor is malfunction by rolling over. A problem of the infrared sensor is a large number of bets malfunction difficulty of installation ${ }^{(3)}$.

In this study, we aim to develop system to focus on caregiver burden and risk of midnight prowl in question in the field of the welfare, to reduce the mental burden of subjects and caregivers. We aim to solve the problem by installing the ceiling Kinect in this system, the use of depth of built-in sensor.

\section{Prowl Prevention System}

\subsection{Processing}

The system is operated by placing the ceiling Kinect sensor. Image of the system was shown in Fig. 1. Procedure of the behavior is in the following order:

i. Acquisition of background information from the depth sensor.

ii. The acquisition of depth information of current.

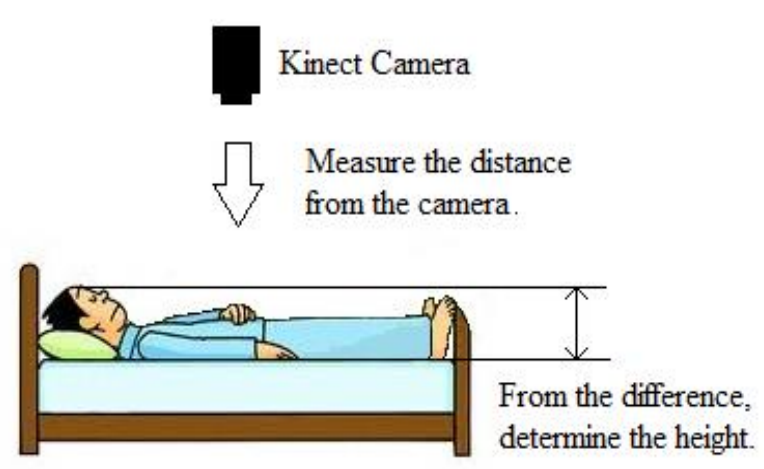

Fig. 1. Image of the system. 


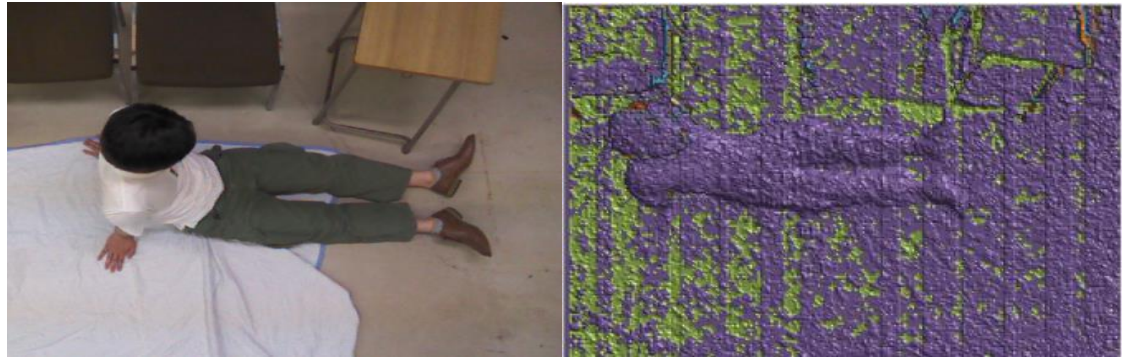

(a) RGB image (b) Difference results

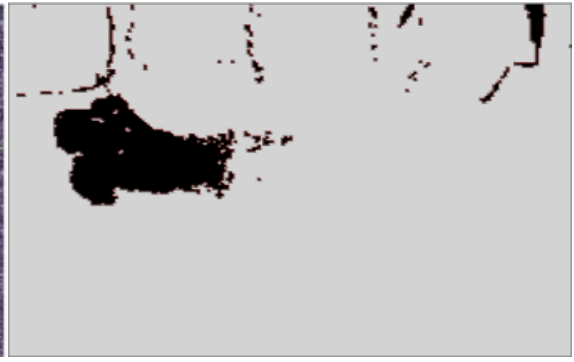

(c) Binary image

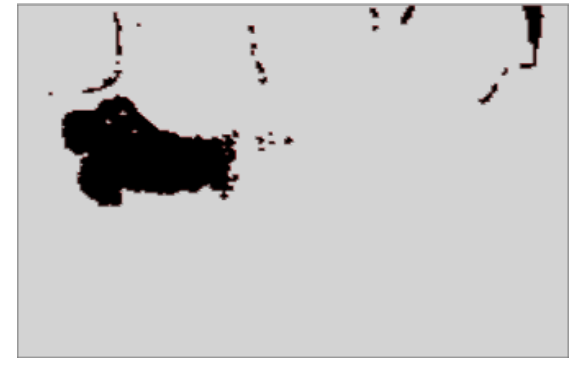

(d) After filtering image

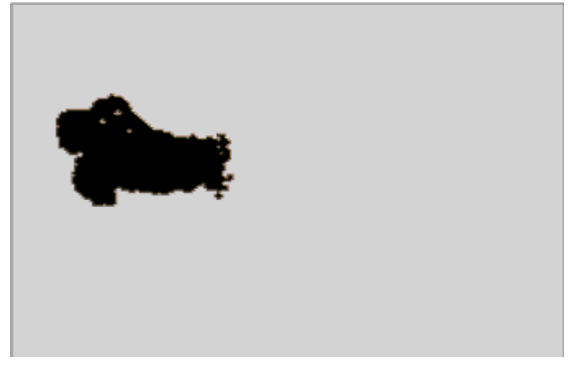

(e) After noise removal image

Fig. 2. Flow of the image processing.

iii. Difference acquisition of depth and background.

iv. Binary processing.

v. Filtering of the image.

vi. Processing labeling.

vii. The removal of the label area equal to or less than the Threshold.

viii. People detection in the height from the threshold.

ix. Tracking of the center coordinates of the person.

x. It is going to bed or leaving the determination of the track at the end.

xi. Decision of leaving or going to bed when ending of tracking.

xii. Performing a state determination of the track at the end.

The flow of image processing shows in Fig. 2.

\subsection{Save Depth Information}

Depth information is given as a string of 16 bits for each pixel from the Kinect. Lower 3 bits is a player identification bit, hence the depth actual information will be represented in 13 bits up. Acquisition of the depth data was performed by numeric conversion strings 13 bits by the function. Since the depth data takes a value of 0 when the detection ranges, to convert the maximum value in that case.

\subsection{Difference Acquisition of Depth Information}

This system gets the difference between the depth of background data that has been saved and the depth of the current data. Difference data is the numerical value of the distance from the camera, obtaining the height of the subject by subtracting from the distance value that is a maximum possible of the camera.

\subsection{Human Detection}

The threshold value is set from the data obtained by the difference. In this case, it is detected as those persons having a height equal to or higher than the threshold value. At this time, obtain the coordinates for each pixel in a range of detection.

\subsection{Tracking Center Point}

While detecting a person, the system continues to acquire the coordinates. This system takes the average value for each $\mathrm{x}$-and $\mathrm{y}$-coordinates of the coordinate from the range of detection. This system obtains the center point, and displays a square image ( $\mathbf{\square})$ at the center point. 


\subsection{Decision of the State}

The system determines whether the target went out. If the center point disappears at the edge of the range, this system determines that the target went out of the range, and notifies the administrator it. If the center point disappears at the other position, this system determines that the target lain down in the range. While the center point is present, the system determines that the target is in the range.

\section{Operation Verification}

In this chapter, we tried the test of this system. Kinect is installed on the ceiling of about 2.4 meters in height. Field of view of the camera was about 2 meters in height, and about 2.5 meters width.

\subsection{Method of Verification}

We tried the verification of the following items:

(a) Save Depth Information

The field of view of the camera is separated nine. The depth information of the posture (Standing, Sitting, Upper body raising, Lying, On all fours, Prone posture, Flat on back) of each is saved. To check its value, the depth information is written to text.

(b) Difference Acquisition of Depth Information

The background depth is saved, and the difference between the background and depth of now is obtained. To check its value, the difference between background depth and actual depth are written to text.

(c) The Human Detection in Threshold

The photos and the actual result of processing by the threshold are compared, to verify detecting person using the threshold from the depth.

(d) Center Point Tracking

It is checked whether $\boldsymbol{a}$ is displayed in the center coordinates by set of coordinates of the person. In addition, it is confirmed that the tracking is made to the movement of the target.

(e) Judgment at the End of Tracking

When a person is sleeping state or exits to the outside of the camera, it is tried the operation of the system for each. It is checked that this system warns when a person exits to the outside of the camera.

\subsection{Verification Result}

About process i iii, the results are shown in Fig. 1. As shown in the Fig. 3, system succeeded to detects the height of the object from the difference result. From these

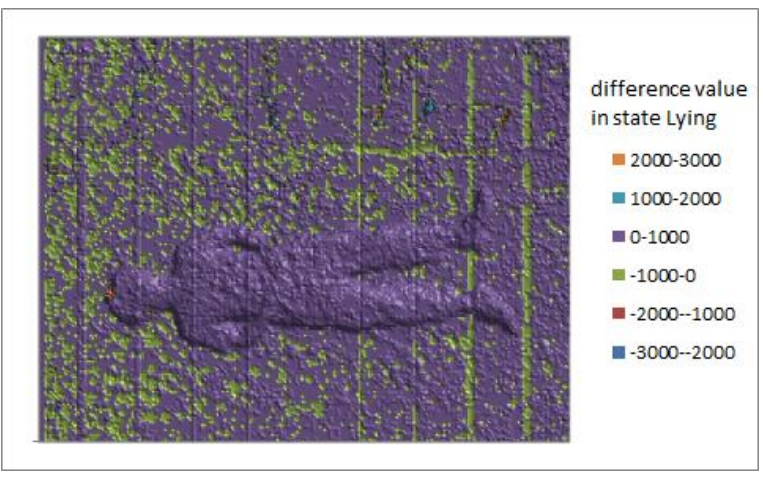

(a) Difference result in laying

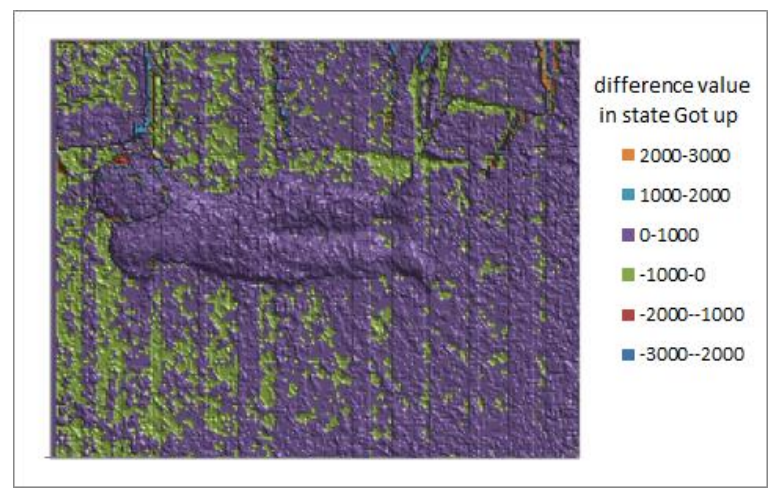

(b) Difference result in state got up

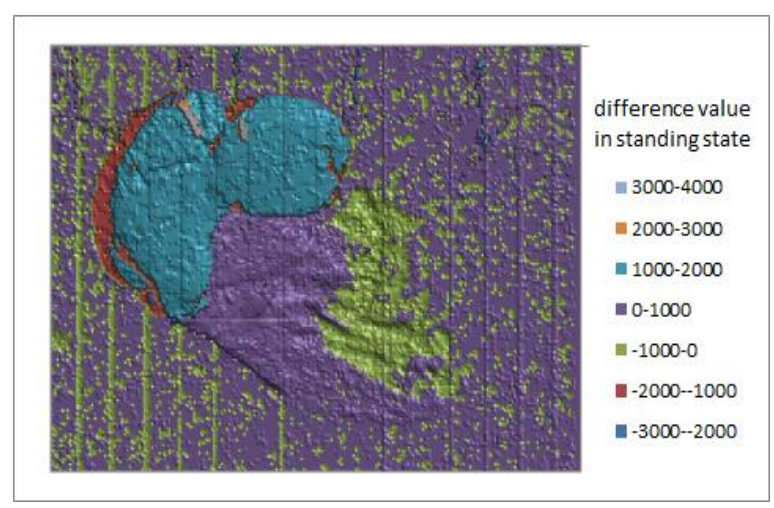

(c) Difference result in standing state

Fig. 3. Difference results.

result, we was set to 500 the threshold for determining a state got up and state lying down. About process iv viii, the actual picture and flow of the results of the processing was shown in Fig. 4. The system performed by the determination threshold value as shown in the figure, it is possible to obtain the center coordinates by performing the determination of a person after removing noise, and it was possible to detect the get up. Further, a does not appear for have been removed by the threshold value in the state 


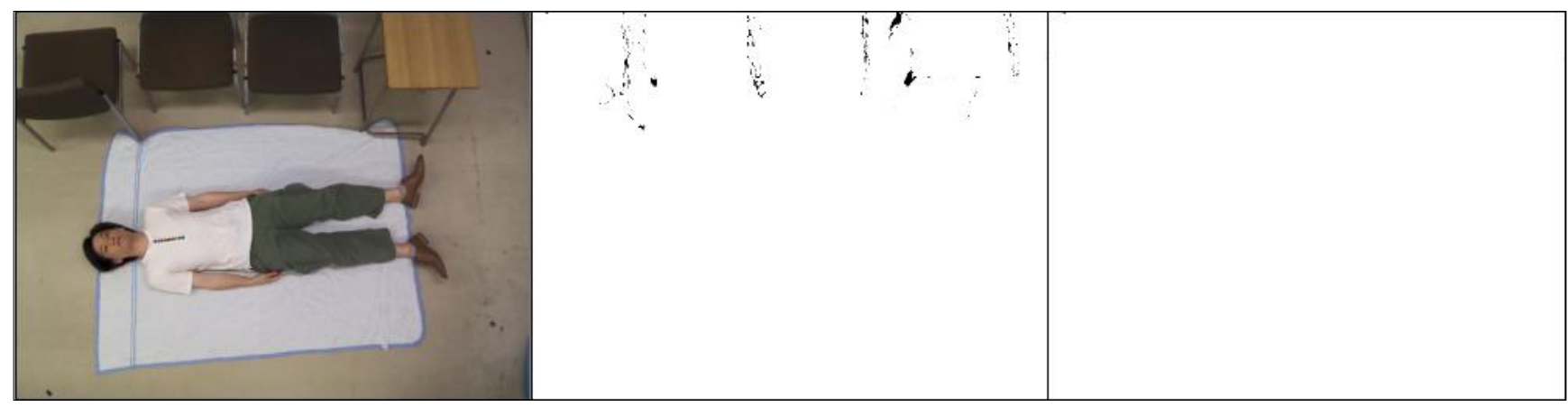

(a) laying

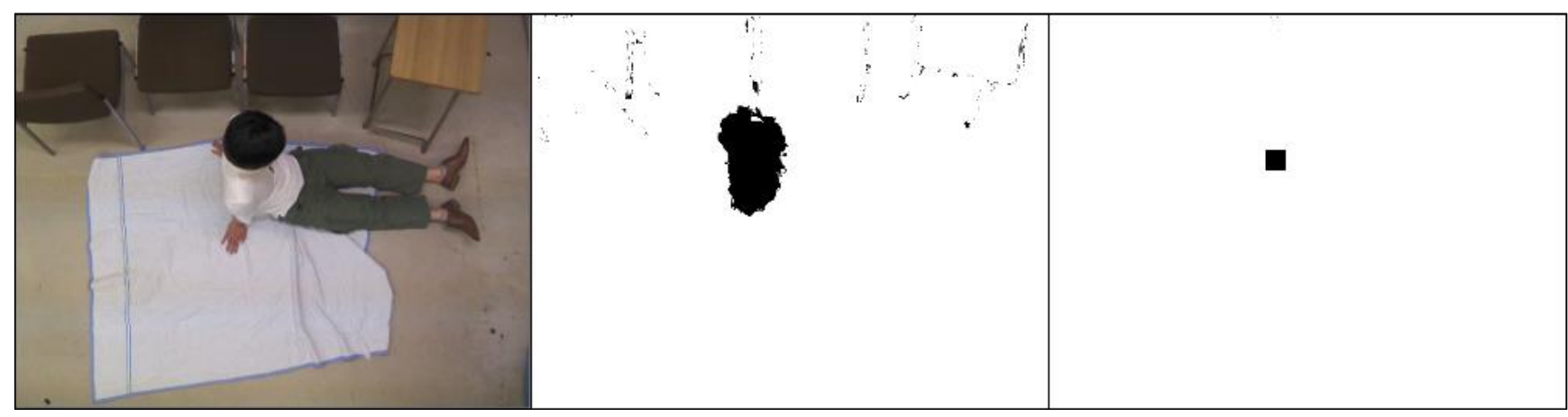

(b) Got up

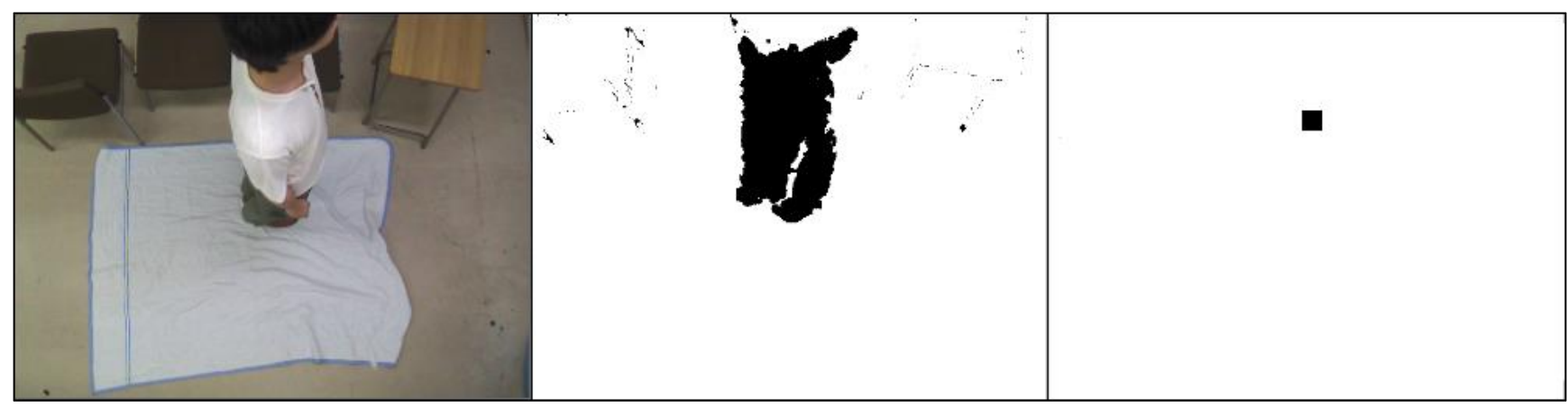

(c) Standing

Fig. 4. System execution result.

laying. And about process $\mathrm{x}$ and $\mathrm{xi}$, the state of the processing results of exit detection and tracking of state in each location was shown in Fig. 5. As shown in the figure, it was confirmed that system works as intended. Determining the center point obtained from the processing results by taking the average of coordinates corresponding, and confirmed the system has been performed.

\section{Conclusions}

In this study, after considering the issues of existing system to prevent midnight prowl, we developed a system that can solve these problems, and demonstrate the operation. From verification result, we were able to confirm the operation of detection of the person and tracking.

Although could be confirmed operation of system from the experimental, system tracking was limited to one subject. There is a need to allow the tracking of each even if multiple people were reflected to enhance the versatility of the system. 


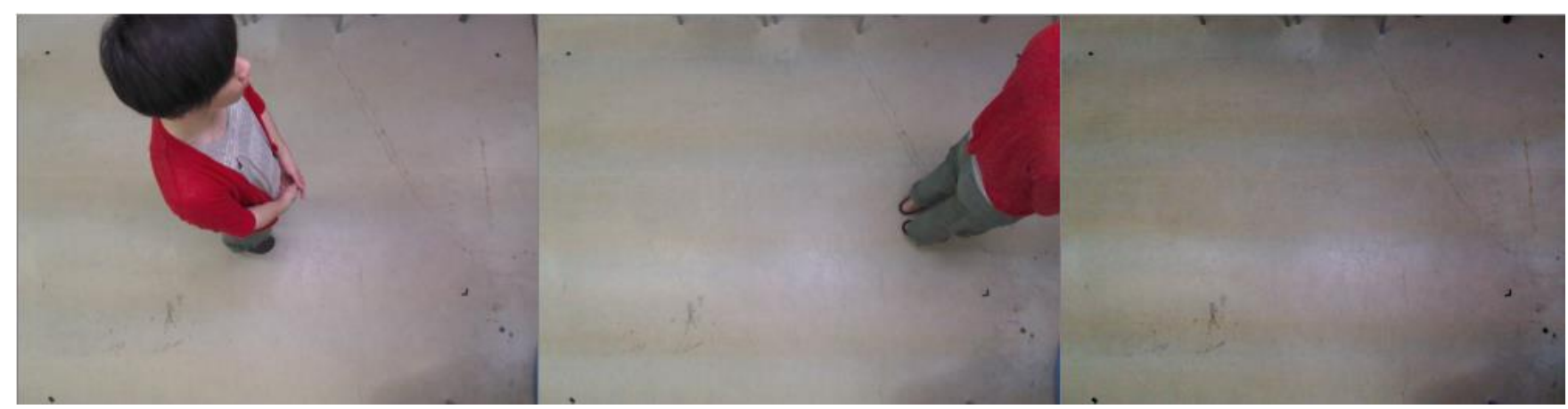

(a) RGB Image

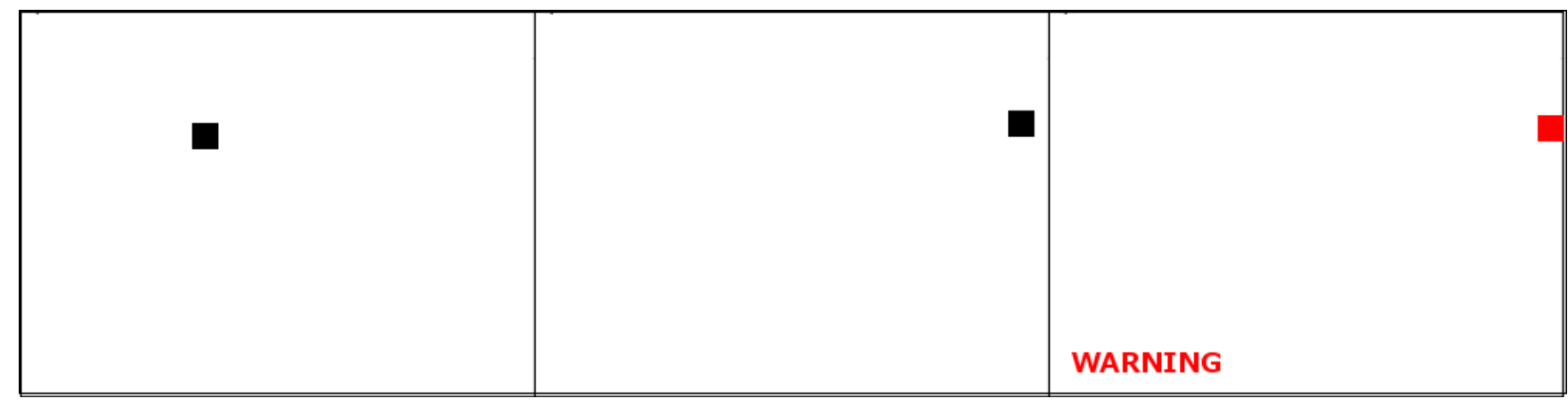

(b) Tracking Image

Fig. 5. Track results by the system.

\section{References}

(1) Hiroyuki Imazono and Koji Matsuba : "Development of helper support system in aged care facilities", Journal of Kinki Polytechnic University, No. 19, pp. 51-52, 2011

(2) Kentaro Yamazaki : "Outcome base for quality improvement of welfare and nursing care servicesImplementation of PDCA cycle from the actual situation evaluation - study on the actual condition of the elderly wandering death case", Summary-sharing research report, pp. 138-146, 2012

(3) Yasuko Fujii, Junko Nishimura, Noriko Mitsui, Hiromi Nagasawa, and Satoko Itaya : "Effects of fall prevention measures on: use the out-of-bed sensor "Matta-Kun" in patients with Parkinson's syndrome", Yamaguchi University hospital nursing workshop Acquisition, Number, pp. 11-16, 2001 